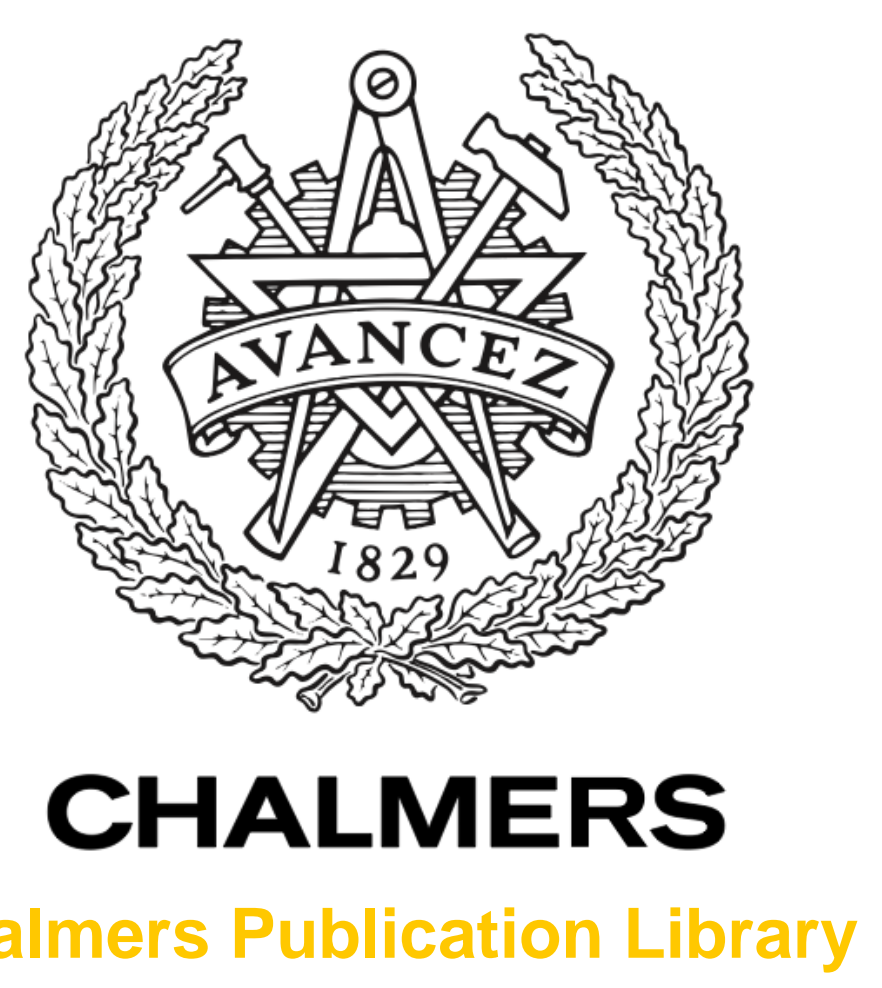

Chalmers Publication Library

\title{
Measurements of relative Throughput in Reverberation Chamber using Universal Software Radio Peripheral
}

This document has been downloaded from Chalmers Publication Library (CPL). It is the author's version of a work that was accepted for publication in:

8th European Conference on Antennas and Propagation, EuCAP 2014, The Hague, The Netherlands 6-11 April 2014

Citation for the published paper:

Einarsson, B. ; Hussain, A. ; Kildal, P. (2014) "Measurements of relative Throughput in Reverberation Chamber using Universal Software Radio Peripheral". 8th European Conference on Antennas and Propagation, EuCAP 2014, The Hague, The Netherlands 6-11 April 2014 pp. 1105 - 1108.

http://dx.doi.org/10.1109/EuCAP.2014.6901963

Downloaded from: http://publications.lib.chalmers.se/publication/203783

Notice: Changes introduced as a result of publishing processes such as copy-editing and formatting may not be reflected in this document. For a definitive version of this work, please refer to the published source. Please note that access to the published version might require a subscription. 


\title{
Measurements of Throughput in Reverberation Chamber using Universal Software Radio Peripheral
}

\author{
Bjarni Pór Einarsson ${ }^{1}$, Ahmed Hussain ${ }^{1}$, Per-Simon Kildal ${ }^{1}$ \\ ${ }^{1}$ Department of Signals and Systems, Chalmers University of Technology, Gothenburg, Sweden, \\ Bjarni Pór Einarsson <raincoat0410@gmail.com>
}

\begin{abstract}
This paper aims to show how Universal Software Radio Peripheral can be used in conjunction with reverberation chamber to measure throughput of wireless communication systems in Rich Isotropic Multipath environment. The results are in agreement with theories based on the threshold receiver model.
\end{abstract}

Index Terms-Universal Software Radio Peripheral, Reverberation Chamber, Rich Isotropic Multipath Channel, Throughput.

\section{INTRODUCTION}

There is an increasing complexity of the commercial digital wireless systems used in today's wireless telecommunications. Therefore, there is an increasing demand for simple and cost effective platforms to measure and quantify the performance of everything from innovative hardware to new algorithms, and even simplified theoretical system models. The Universal Software Radio Peripheral (USRP) is such an inexpensive and versatile platform, which can be used for implementation of various algorithms in order to test their effectiveness in practice [1]. The Reverberation Chamber (RC) accurately emulates the Rich Isotropic Multipath Channel (RIMP), which is a useful reference channel for most modern wireless communication devices [2].

In this paper we want to demonstrate for the first time that USRP and RC together can accurately measure relative system throughput for both simple and complex systems over the RIMP, and that the results are in agreement with theories based on the threshold receiver model presented in [3]. The accuracy of measurements in RC is well proven [4] and shown to be in agreement with results obtained in anechoic chambers for passive devices [5]-[6]. In addition, the RC can emulate different time delay spreads [7], and thereby it is possible to measure active devices and complete systems. The measured results are in good agreement with theory based on the threshold receiver model in [3], by which even the effect of OFDM in the LTE system for different time delay spreads can be modelled [8]. The threshold receiver has also been extended to the MIMO multiplexing case [9]

\section{THEORY}

The threshold receiver model for the AWGN channel in terms of group error rate (GER) can be expressed as (1)

$$
\operatorname{GER}(P)= \begin{cases}1, & P<P_{t} \\ 0, & P>P_{t}\end{cases}
$$

where $\mathrm{P}$ is the received power and $\mathrm{P}_{\mathrm{t}}$ is the system threshold power. This can be extended to include more complex systems and then takes the notation of (2)

$$
\operatorname{GER}_{a v}\left(P_{a v}\right)=\operatorname{CDF}\left(\frac{P_{t}}{P_{a v}}\right)
$$

$$
\text { Throughput }=\text { Maxrate } *\left\{1-\operatorname{GER}_{a v}\left(P_{a v}\right)\right\}
$$

where $\mathrm{P}_{\mathrm{av}}$ is the average received power and the cumulative distribution function (CDF) in (2) is the channel CDF after the MIMO and OFDM processing. We can then use (3) as a general expression for throughput where the probability density function or CDF for the channel is known in addition to the MIMO and OFDM algorithms. The channel is well known for the RIMP to have Rayleigh distribution, and we assume in our works the theoretical signal-processing models for OFDM and MIMO based on the above threshold receiver model as used in [3], [8] and [9].

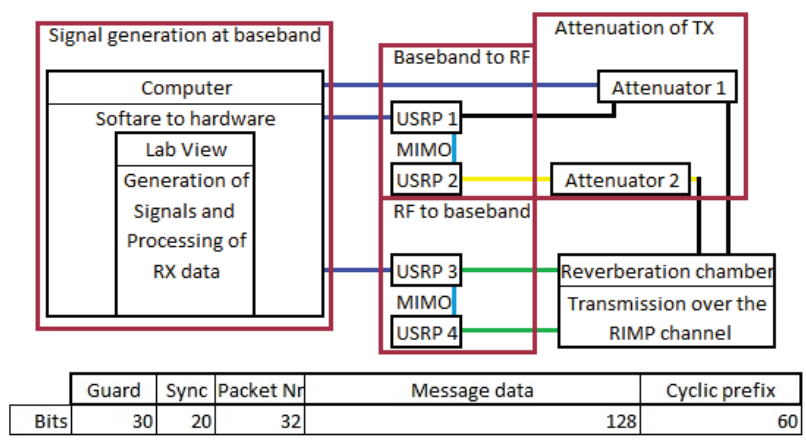

Fig. 1 System diagram and packet setup

\section{SYSTEM PARAMETERS}

The system is set to operate at relatively low throughput and center frequency compared with what can be achieved by the USRP platform, in order to give a larger margin for error on packet and frame detection. The sampling rate is set at $400 \mathrm{kHz}$ and 8 samples per symbol giving a max symbol rate of $50 \mathrm{k}$ symbols $/ \mathrm{sec}$.

The selected modulation is quadrature phase shift keying (QPSK) with a grey code coding scheme without any error control coding. The chosen pulse shaping filter was root raised cosine of length 6 with roll off factor, $\alpha=0.5$, giving the system a total bandwidth of $75 \mathrm{kHz} \quad\left(\mathrm{B}_{\text {system }}\right)$ during transmission. $\mathrm{B}_{\text {coherence }}$ was $3.5 \mathrm{MHz}$ for the present setup in the reverberation chamber. This means that the channel is flat fading because $\mathrm{B}_{\text {system }}<<\mathrm{B}_{\text {coherence }}$. 


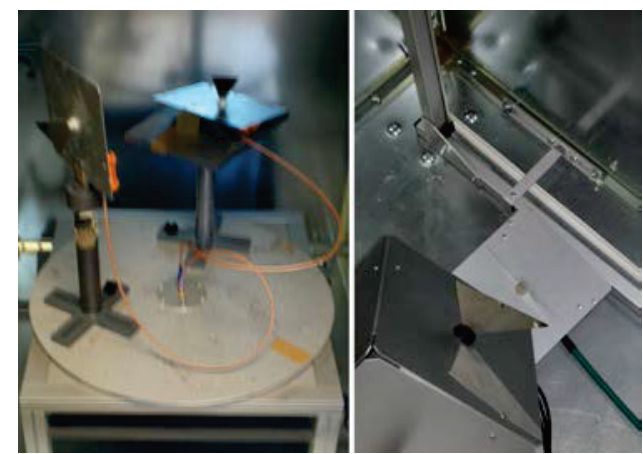

Fig. 2 Antenna placement in reverberation chamber(RX left, TX right)

The operating frequency was chosen to be $915 \mathrm{MHz}$ because previous SISO tests had been done at that frequency. For the tests the wideband triangle antennas used as wall antennas in the chamber were used both for transmission and reception, with the antenna placement (Fig. 2) so that there is no correlation between them.

The achieved bitrate $(35 \mathrm{~kb} / \mathrm{s})$ is low due to large packet overhead, as seen in Fig. 1. The results are presented in terms of relative bitrate. This packet configuration is chosen because it was the pre-designated packet configuration in the initial example presented in [9]

All these settings are defined in the LabView code. After defining this, the same code module then constructs the packets for transmission, sends them at baseband to the USRPs which then modulate them to radio frequency (RF) and transmit them. Upon reception the received message is demodulated from RF to baseband and then sent back to the LabView code, which processes the received packet and determines the throughput.

For each step of the attenuator a large amount of packets was chosen for better relative accuracy. In total the chosen text broke down to 842 packets. These are then sent 40 times at each step for a total of 33380 packets.

\section{MEASUREMENT SETUP}

Initial single input single output (SISO) over the air (OTA) and conducive measurements were performed using a pair of USRPs connected to a control computer. With the transmitter (TX) port of one USRP connected to the input of the digital attenuator (DA) and the output of the DA either connected to the receiving (RX) USRP for conductive measurements or the input port of the RC for OTA measurements.

The power received was carefully calibrated for each attenuation step of the DA. This was done by measuring it at the connection to the RX with a spectrum analyzer, following that the average path loss was carefully calibrated for the RC and the connecting cables. For OTA measurements the path loss of the RC was subtracted (in $\mathrm{dB}$ ) from the known transmitted power for each attenuation step.

For the single input multiple output (SIMO) and multiple input single output (MISO) measurements the setup was the same as for SISO. But, we used a third USRP connected to another USRP via a multiple input multiple output (MIMO) cable. This third USRP had the same characteristics as the other two. During the SIMO OTA measurements this was simply connected to the second output port of the RC, and for MISO OTA the DA was connected to the RX instead of the TX.

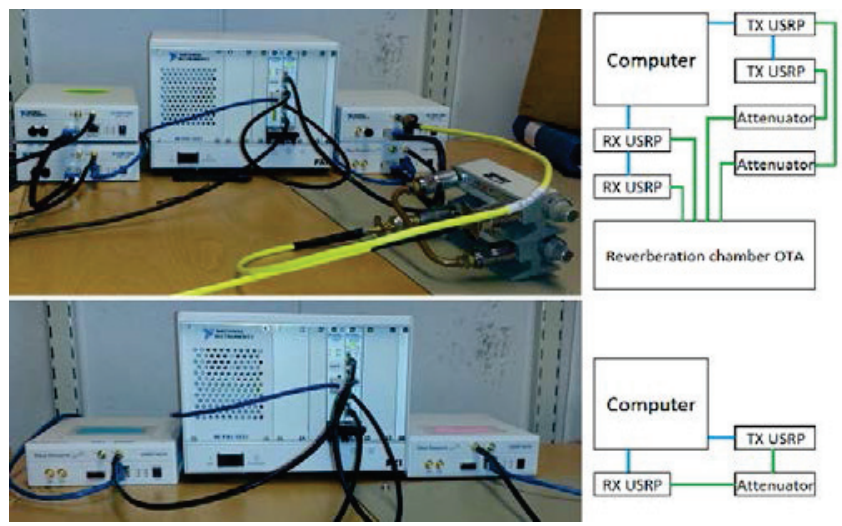

Fig. 3 MIMO OTA and conductive measurement setup

For the MIMO measurements a fourth TX USRP was added to the setup and connected to the standalone USRP via MIMO cable. Its output was then connected to a manual attenuator which was then connected to the second TX port of the RC (Fig. 1, Fig. 3). The attenuators were calibrated so that they had on average $0.2 \mathrm{~dB}$ offset in TX power between antennas inside the RC

\section{RESULTS}

We see from Figs. 4-7 that there is very good agreement between the measured results and the theoretical results. In most data points there is less than $1 \mathrm{~dB}$ offset between the theoretical throughput and achieved relative throughput of the system.

We see that the measured results agree well with theoretical values for the simple case of SISO \& conductive (Fig. 4) and the more complex case of SIMO with selection combining (SC) and maximum ratio combining (MRC) (Fig. 5).

In Fig. 6. We see also for the MISO case that there is an overall good agreement between the measured and theoretical results.

We see in Fig. 7 that the measured throughput data shows a good agreement with the theoretical throughput for the $2 \times 2$ MIMO as well. All curves are for the single bit stream case, so we have not yet tested MIMO multiplexing from which two bit streams can be achieved. 


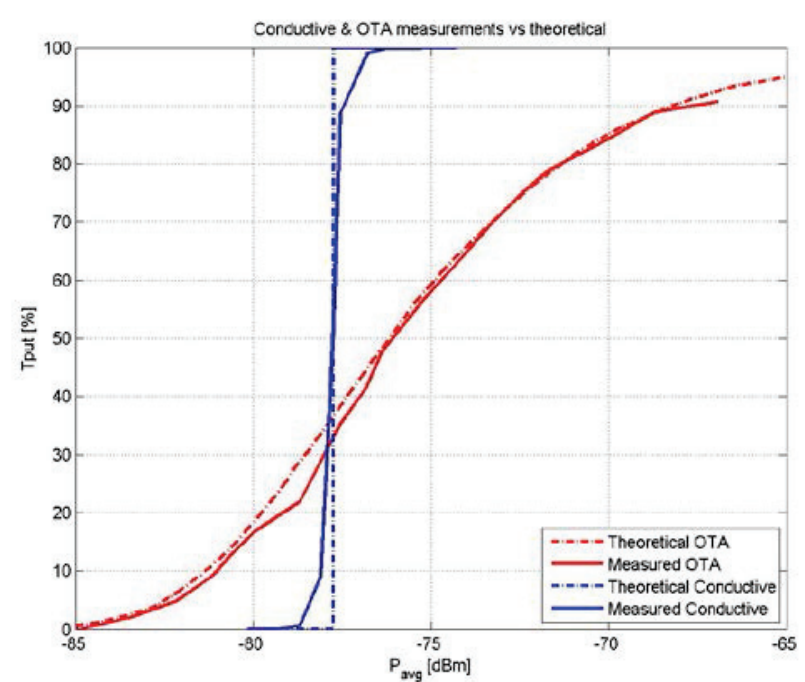

Fig. 4 Theoretical vs. measured values for SISO system

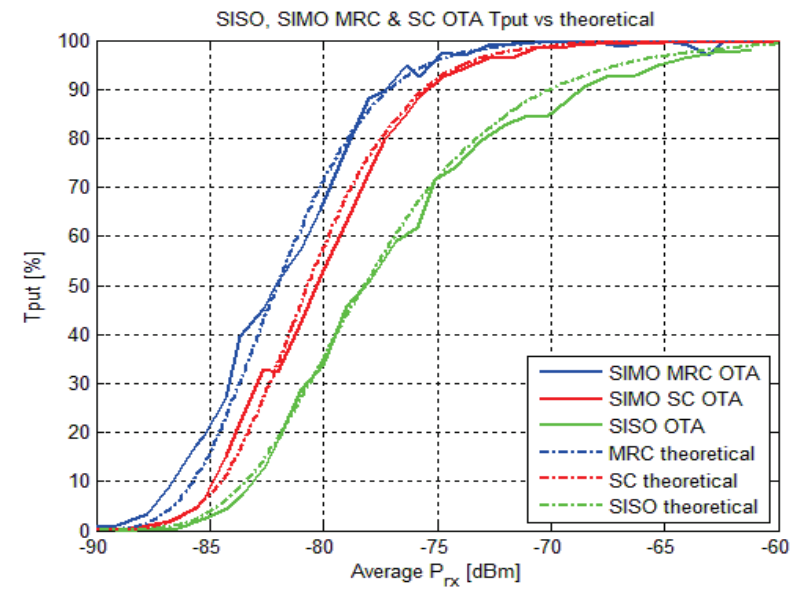

Fig. 5 Theoretical vs. measured values for $1 \times 2$ SIMO \& SISO systems

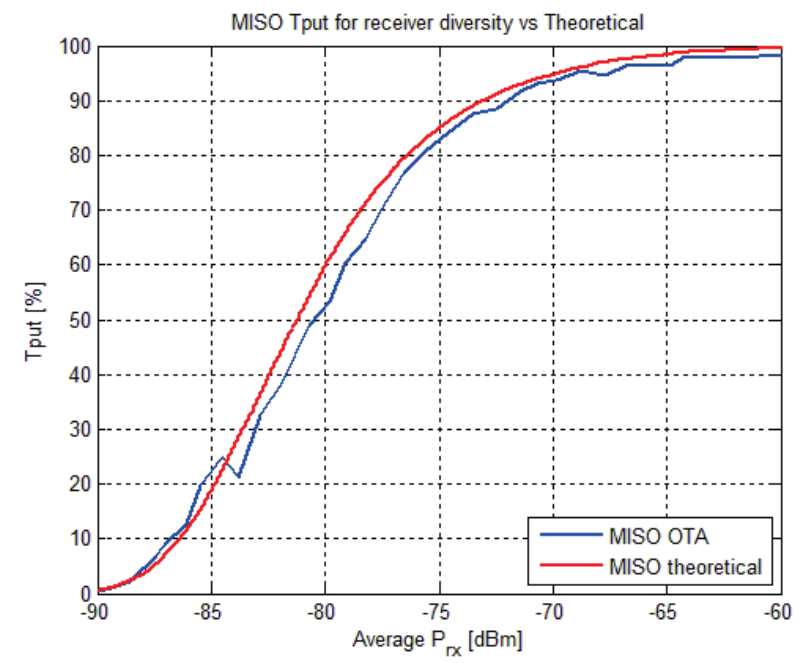

Fig. 6 Theoretical vs. measured values for 2x1 MISO system

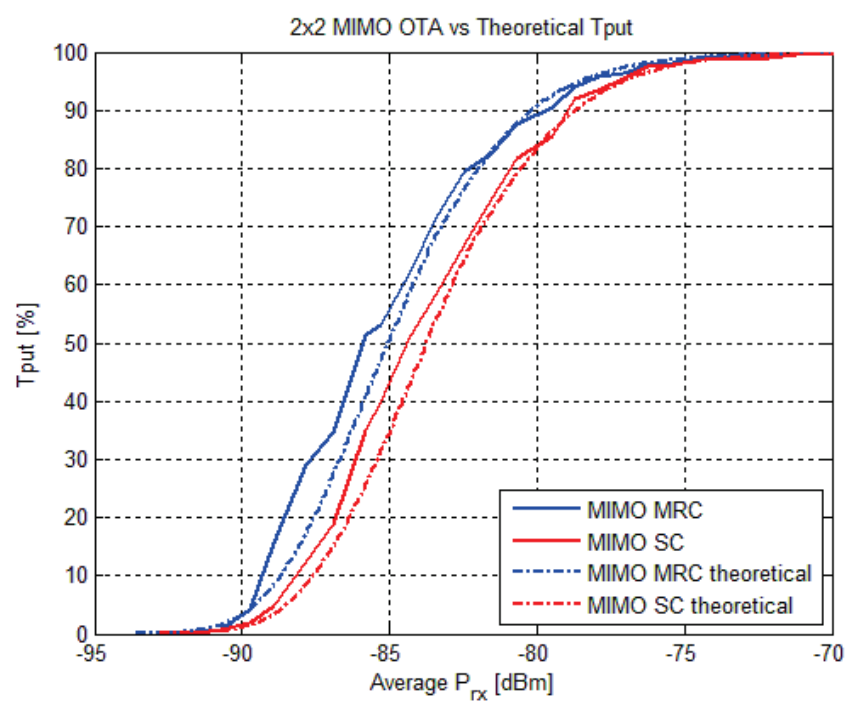

Fig. 7 Theoretical vs. measured values for 2x2 MIMO system with TX and RX diversity.

\section{LIMITATIONS OF THE USRP}

The USRP platform provides a simple and cheap tool for the measurement and simulation of radio communication systems of varying complexity, but it is not without limitations.

The system can without problems transmit and measure continuous data streams both conducted and OTA with good precision and speed. This is given that the generation of the packet is done beforehand and processing of the received data is done offline.

However, the system struggles with online processing of the data in real time and usage of coding schemes that rely on channel state information at the TX side with more than one TX USRP connected with a MIMO cable. This is due to a initialization time $(120 \mathrm{~ms})$ that seems to be required to initialize the TX pair over the MIMO cable.

Online processing of the data requires computer with a high processing speed, and efficient computation algorithms in order to avoid large wait times between transmissions.

The USRP system suffers from a significant amount of crosstalk between TX and RX channels. Therefore, even if a single USRP is capable of acting both as TX and RX, the results will not be usable for precision measurements, because the crosstalk will alter the results significantly.

Another thing worth noting is that the threshold power for the conductive measurements appears to be heavily affected by the driver version of the USRP platform. When we updated the driver, the threshold power was improved, and this should theoretically not be possible. 


\section{CONCLUSIONS}

We have presented results from measuring data rate throughput in reverberation chamber using USRP. This has been done using a variety of single bit stream cases in the range of SISO up to $2 \times 2$ MIMO. The basic combining methods of SC and MRC were used. All results can be explained by signal processing models and using the threshold receiver model. Thus, we have found that the USRP is a viable low cost platform for measuring communication systems, providing a useful tool for researchers to implement and test their own algorithms for modulation, error coding and MIMO in a practical measurement setup.

\section{REFERENCES}

[1] http://www.ni.com/usrp/

[2] P.-S. Kildal, C. Orlenius, J. Carlsson, "OTA Testing in multipath of antennas and wireless devices with MIMO and OFDM", Proceedings of the IEEE, Vol. 100, No. 7, July 2012

[3] P.-S. Kildal, A. Hussain, X. Chen, C. Orlenius, A. Skårbratt, J. Åsberg, T. Svenson, T. Eriksson, "Threshold receiver model for throughput of wireless devices with MIMO and frequency diversity measured in reverberation chamber" IEEE Antennas Wireless Propag. Lett, vol. 10, pp. 1201-1204, 2011
[4] P.-S. Kildal, X. Chen, C. Orlenius, M. Franzén, C. Lötbäck Patané, "Characterization of Reverberation Chambers for OTA Measurements of Wireless Devices: Physical Formulations of Channel Matrix and New Uncertainty Formula", IEEE Transactions on Antennas and Propagation, Vol. 60, No. 8, pp. 3875-3891, Aug. 2012.

[5] X. Chen, P. Kildal, J. Carlsson, and Y. Jian, "MRC Diversity and MIMO Capacity Evaluations of Multi-Port Antennas Using Reverberation Chamber and Anechoic Chamber," Antennas and Propagation, IEEE Transactions on, vol. 61, pp. 917-926, 2013.

[6] X. Chen, P.-S. Kildal, J. Carlsson, J. Yang, "Comparison of Ergodic Capacities from Wideband MIMO Antenna Measurements in Reverberation Chamber and Anechoic Chamber", Antennas and Wireless Propagation Letters, vol. 10, pp. 446-449, May 2011.

[7] C. Xiaoming, P. S. Kildal, C. Orlenius, and J. Carlsson, "Channel Sounding of Loaded Reverberation Chamber for Over-the-Air Testing of Wireless Devices: Coherence Bandwidth Versus Average Mode Bandwidth and Delay Spread," Antennas and Wireless Propagation Letters, IEEE, vol. 8, pp. 678-681, 2009.

[8] A. Hussain and P. Kildal, "Study of OTA throughput of LTE terminals for different system bandwidths and coherence bandwidths," in Antennas and Propagation (EuCAP), 2013 7th European Conference on, 2013, pp. 312-314.

[9] X. Chen, P.-S. Kildal, and M. Gustafsson, "Characterization of implemented algorithm for MIMO spatial multiplexing in reverberation chamber," IEEE Transactions on Antennas and Propagation, vol. 61, no. 8, pp. 4400-4404, August. 2013.

[10] https://decibel.ni.com/content/docs/DOC-18801 